(C) 1998 OPA (Overseas Publishers Association) N.V.

Published by license under the Gordon and Breach Science Publishers imprint. Printed in India.

\title{
RF Performance of Si/SiGe MODFETs: A Simulation Study
}

\author{
S. ROY, A. ASENOV*, S. BABIKER, J. R. BARKER and S. P. BEAUMONT \\ Nanoelectronics Research Centre, Department of Electronics and Electrical Engineering, \\ Glasgow University, Glasgow G12 8LT, Scotland, UK
}

\begin{abstract}
The microwave performance potential of $\mathrm{Si} / \mathrm{SiGe}$ pseudomorphic MODFETs are studied, in comparison to state of the art InGaAs pseudomorphic HEMTs. Both devices have equivalent structures corresponding to a physical HEMT used for calibration. We use an RF analysis technique based on transient Monte Carlo simulations to estimate the intrinsic noise figures, the RF figures of merit $f_{\mathrm{T}}$ and $f_{\max }$, and the effect of contact and gate resistances. Both devices exhibit velocity overshoot below the gate region. It is shown that the difference in noise figures and $f_{\mathrm{T}}$ values can be mainly attributed to differences in device channel velocity. $f_{\max }$ exhibits a strong dependence on device contact resistance, eroding some of the performance advantage of the pseudomorphic HEMT.
\end{abstract}

Keywords: $\mathrm{Si} / \mathrm{SiGe}$, heterostructures, Monte Carlo, microwave performance, RF analysis

\section{INTRODUCTION}

Recent theoretical and experimental studies show that low field mobility and velocity overshoot are enhanced in Si layers grown pseudomorphically on relaxed $\mathrm{SiGe}$ substrates. Induced strain breaks the six-fold degeneracy of the $\mathrm{Si}$ conduction band, resulting in an improved band offset for the two conduction valleys whose transverse effective mass is in the plane of the heterojunction. This increases the in plane effective mobility and reduces intervalley scattering in the Si layer [1]. Modulation doped field effect transistors (MODFETs) based on this material system have been demonstrated, and show significant potential for RF applications $[2,3]$. Although measured mobilities are lower than those reported in optimal III-V based devices, the compatibility between $\mathrm{SiGe}$ and conventional Si processing technology makes such MODFETs attractive for Si MMICs design and microwave signal processing applications integrated on conventional Si chips. Therefore, comparison of strained Si channel devices with well established members of the III - V family will

\footnotetext{
*Corresponding author. Tel.: ++44 141330 5233, Fax: ++44 141330 4907, e-mail: A. Asenov@elec.gla.ac.uk.
} 
provide greater insight into their potential performance and limitations.

As part of this goal, we here study the microwave performance potential of a $\mathrm{Si} / \mathrm{SiGe}$ pseudomorphic MODFET in comparison with a state of the art InGaAs channel pseudomorphic HEMT, using an RF analysis technique based on transient Monte Carlo simulations.

\section{DEVICE STRUCTURES AND PERFORMANCE ANALYSIS}

To allow a fair comparison similar device layer structures are considered (Figs. 1a,b). Both devices have $\delta$-doping separated by a $2.5 \mathrm{~nm}$ spacer from the channel, a $T$ shape recess gate with $50 \mathrm{~nm}$ recess offset and heavily doped ( $n$-type $10^{18} \mathrm{~cm}^{-3}$ ) cap layers. An effective $\delta$-doping of $5 \times 10^{12} \mathrm{~cm}^{-2}$ is considered in the both cases. Total gate-to-channel separation is $22 \mathrm{~nm}$ and the gate length is $0.12 \mu \mathrm{m}$. $p$-type background substrate doping of $10^{14} \mathrm{~cm}^{-3}$ is considered. This corresponds to the dimensions of real pseudomorphic HEMT fabricated at the Glasgow Nanoelectronics Research Centre and used for validation and calibration of the RF Monte Carlo analysis technique (described in an accompanying paper [4]). We realise that for the $\mathrm{Si} / \mathrm{SiGe}$ MODFET this may pose unresolved growth problems. Specifically we conjecture im-

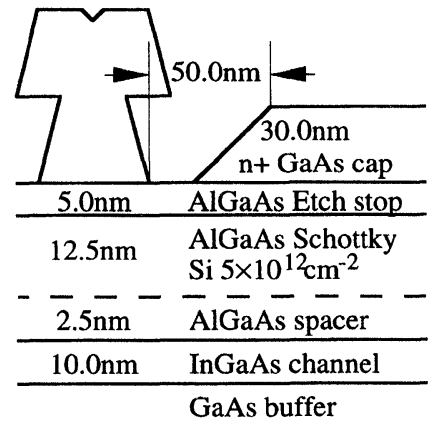

(a)

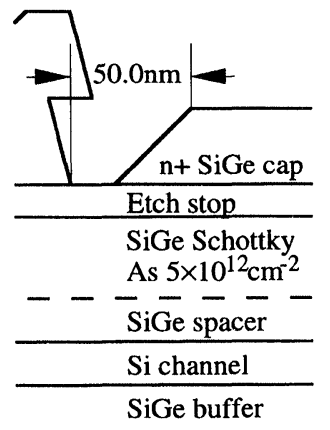

(b)
FIGURE 1 Vertical layer structure of (a) a $0.12 \mu \mathrm{m}$ pseudomorphic HEMT and (b) a $0.12 \mu \mathrm{m}$ pseudomorphic MODFET. Both devices have the same vertical dimensions. provements in growth technology and low temperature processing to allow formation of a well defined As $\delta$-doping supply layer. For simplicity of Monte Carlo modelling a uniform SiGe substrate is assumed, instead of a 'virtual substrate' with graded Ge concentration. The 'etch stop' region of the $\mathrm{Si} / \mathrm{SiGe}$ device is also considered as (undoped) SiGe. Simple 1-D Poisson calculations indicate that if a strained $\mathrm{Si}$ 'etch stop' region is included, it will exhibit negligible parallel conductance at a d.c. bias of $V_{\mathrm{G}}=-0.25 \mathrm{~V}$.

RF analysis of HEMT and MODFET performance is based on Monte Carlo simulation of device transient response. We follow Yamada's [1] treatment of the effect of the strain on the $\mathrm{Si}$ channel band structure, with conduction band splitting of $\Delta E_{\mathrm{c}}=0.67 x(\mathrm{eV})$ and band gap $E_{\mathrm{g}}=$ $1.11-0.74 x(\mathrm{eV})$. However the six phonon scattering model of Jacoboni [5] is implemented, instead of the four phonon model in [1]. Acoustic and ionised impurity scattering modes are also included, with ionised impurity scattering calculated from the Brooks-Herring model [6]. Bulk velocityfield characteristics obtained from the model are in agreement with experimental unstrained $\mathrm{Si}$ data at $77 \mathrm{~K}$ and $300 \mathrm{~K}$ and previous results for strained $\mathrm{Si}[1,7]$. The transient Monte Carlo simulations begin by following $5 \times 10^{4}$ superparticles for $2 \mathrm{ps}$ settling time at d.c. bias, then a further $2.0 \rightarrow 2.6 \mathrm{ps}$ during which device statistics are recorded at $1 \mathrm{fs}$ intervals. A step change $\Delta V_{\mathrm{G}}=0.2 \mathrm{~V}$, or $\Delta V_{\mathrm{D}}=$ $0.3 \mathrm{~V}$ is then consecutively applied, and the transient response measured for a further $2 \mathrm{ps}$. It is found that structure and doping dependant $\mathrm{THz}$ oscillations in device drain current (possibly due to plasma oscillations in the channel, or in the heavily doped cap layer) may mask the detailed form of the transients, and so a number of traces are averaged to define the response. Complex $y$-parameters are derived by Fourier transforming these terminal current transients, and used to extract the small signal equivalent circuit, intrinsic noise figures, and estimate the RF performance figures of merit $f_{\mathrm{T}}$ (cutoff frequency) and $f_{\max }$ (maximum frequency of oscillation). Finally, the small signal equivalent 
circuit is augmented by the addition of external impedance, and thus the effect of contact and gate resistance on the 'real' device operation estimated. A detailed description of the analysis process is given in [4].

\section{RESULTS AND DISCUSSION}

The average velocity in the channel of both devices is compared in Figure 2 for a d.c bias of $V_{\mathrm{D}}=1.5 \mathrm{~V}$ and $V_{\mathrm{G}}=-0.25 \mathrm{~V}$. In the pseudomorphic HEMT this corresponds to the region of maximum transconductance, while in the pseudomorphic MODFET it is the region of maximum transconductance achieved while constraining parallel conduction in the $\delta$-doped region to less than $10 \%$ of total conduction. The gate extends from $x=-0.12 \rightarrow 0.0 \mu \mathrm{m}$. Both devices show distinct overshoot below the gate region. Peak velocity in the pseudomorphic MODFET, at the drain end of the strained $\mathrm{Si}$ channel, approaches twice the saturation velocity of bulk unstrained $\mathrm{Si}$. The

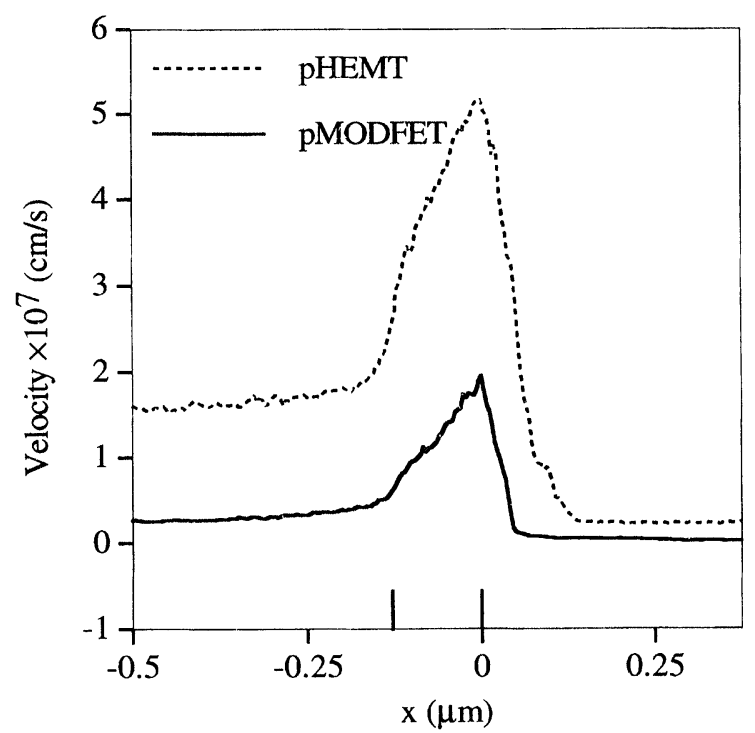

FIGURE 2 Comparison between average velocity in the channel of $0.12 \mu \mathrm{m}$ pseudomorphic HEMT and MODFET. $V_{\mathrm{D}}=1.5 \mathrm{~V}$, with $V_{\mathrm{G}}=-0.25 \mathrm{~V}$ corresponding to maximum transconductance. ratio between peak velocity in the MODFET compared to that of the pseudomorphic HEMT InGaAs channel is 2.8 .

Figures 3-5 and Table I characterise the RF performance of the two devices. The intrinsic noise figures NF (in decibels) are shown in Figure 3. From the definition of NF we postulate that the significant difference between the devices will be in their transconductance $g_{\mathrm{mo}}=Y_{21}$. All other terms $-y$ parameters and current fluctuations $\left\langle\delta I^{2}\right\rangle$ - we therefore represent by an approximate constant $\mathrm{C}$.

$$
N F=1+\left[\frac{Y_{11}}{Y_{21}}\right]^{2} \frac{\left.<I_{\mathrm{d}}^{2}\right\rangle}{\left.<\delta I_{\mathrm{g}}^{2}\right\rangle} \approx 1+\left(\frac{C}{g_{\mathrm{mo}}}\right)^{2}
$$

From Figure 3 and equation (1) the ratio of transconductances $g_{\text {HEMT }} / g_{\text {MODFET }}$ is indeed found to be approximately constant below 60 $\mathrm{GHz}$, with value 2.6. The transconductance values directly obtained from the Monte Carlo simulations are $g_{\text {HEMT }}=87$ and $g_{\text {MODFET }}=31$, with ratio 2.8. It can be concluded that the bulk of the

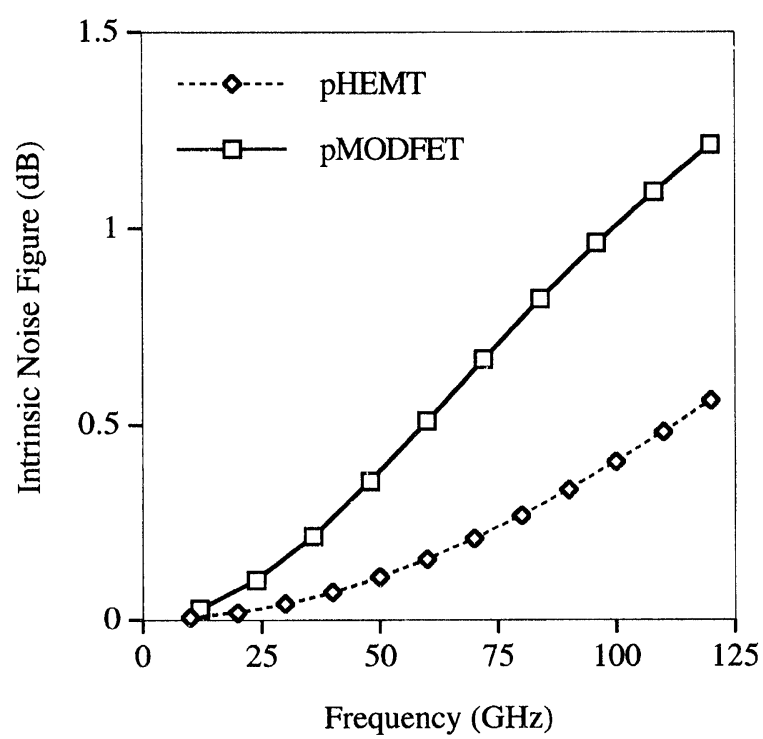

FIGURE 3 Comparison between minimum intrinsic noise figures for pseudomorphic HEMT and MODFET as a function of frequency. $V_{\mathrm{D}}=1.5 \mathrm{~V}$, with $V_{\mathrm{G}}=-0.25 \mathrm{~V}$. 


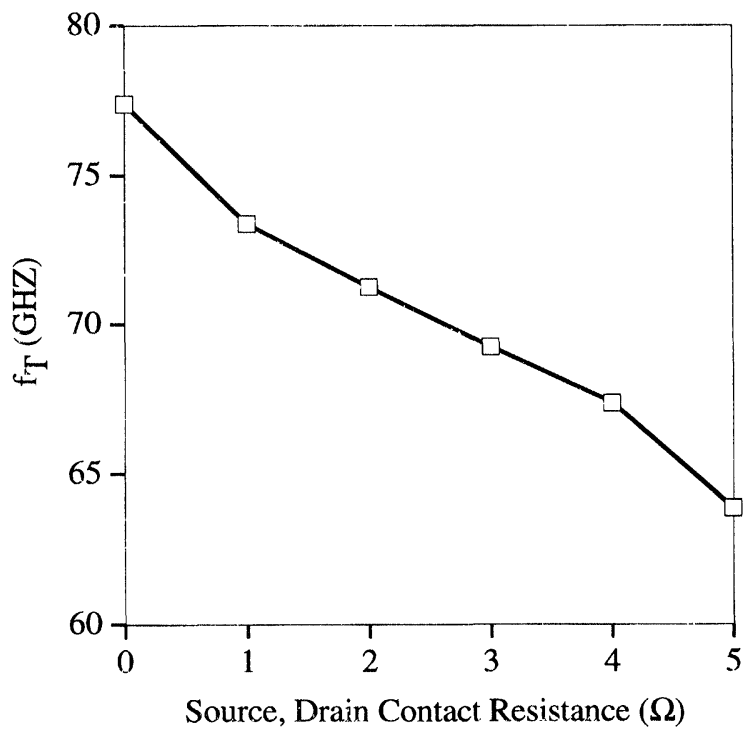

FIGURE 4 Variation of $f_{\mathrm{T}}$ in a pseudomorphic MODFET as a function of source and drain contact resistance ( $f_{\mathrm{T}}$ invariant to changes in gate resistance).

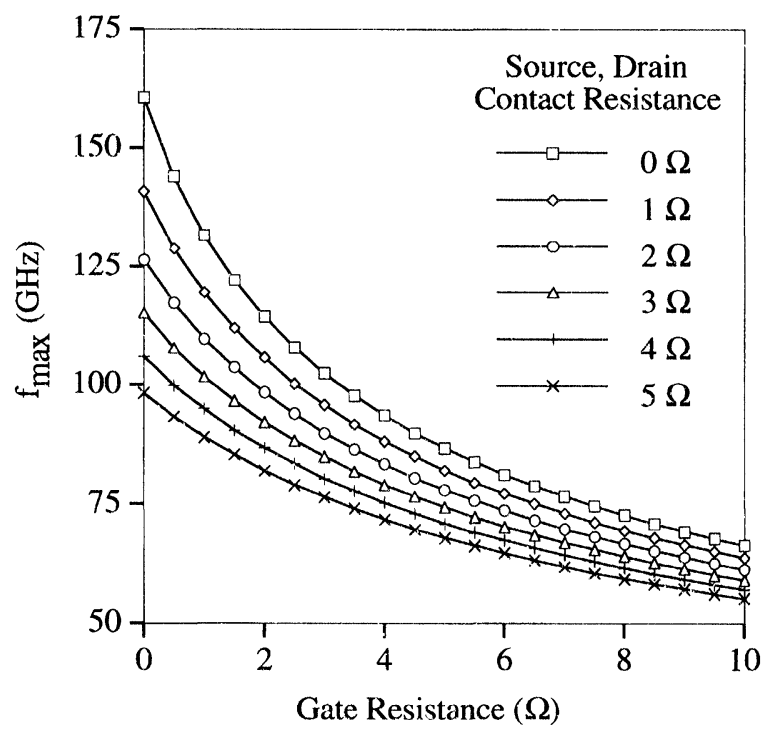

FIGURE 5 Variation of $f_{\max }$ in a pseudomorphic MODFET as a function of gate and contact resistances.

difference in noise figures between the two devices can be attributed to the differences in transconductance - and thus to differences in channel velocity.
TABLE I Calculated RF figures of merit for pseudomorphic HEMT and MODFET at bias $V_{\mathrm{D}}=1.5 \mathrm{~V}, V_{\mathrm{G}}=-0.25 \mathrm{~V}$. Device width of $100 \mu \mathrm{m}$ is assumed. $f_{\mathrm{T}}, f_{\max }$ calculated for both negligible external gate and drain contact resistance, and for more realistic contact resistances of $5 \Omega$

\begin{tabular}{lcc}
\hline $\begin{array}{l}\text { RF figure of } \\
\text { merit (GHz) }\end{array}$ & $\begin{array}{c}\text { Pseudomorphic } \\
\text { HEMT }\end{array}$ & $\begin{array}{c}\text { Pseudomorphic } \\
\text { MODFET }\end{array}$ \\
\hline with negligible contact resistance & \\
$f_{\mathrm{T}}$ & 192 & 77.4 \\
$f_{\max }$ & 634 & 161 \\
gate, source \& drain resistances all of $5 \Omega$ & \\
$f_{\mathrm{T}}$ & 156 & 63.9 \\
$f_{\max }$ & 169 & 68.0 \\
\hline
\end{tabular}

Table I lists the RF figures of merit extracted from the transient response of each device. The ratio of cut-off frequencies $f_{\mathrm{T}}$ for the pseudomorphic HEMT and MODFET is approximately 2.5 and independent of external resistance. This implies that the value of $f_{\mathrm{T}}$ is primarily governed by the channel velocity of the respective device. However, the ratio of $f_{\max }$ for the pseudomorphic HEMT and MODFET is 3.9 for intrinsic devices, and drops to 2.5 when external contact resistances of $5 \Omega$ are applied. A more realistic inclusion of device parasitics in this case reduces the advantages of the pseudomorphic HEMT. Finally, Figures 4 and 5 detail the effect of varying contact resistance on both figures of merit for the pseudomorphic MODFET, and show clearly the strong dependence of $f_{\max }$ on device parasitics.

\section{CONCLUSIONS}

The microwave performance potential of a $\mathrm{Si} / \mathrm{SiGe}$ pseudomorphic MODFET was studied, in comparison to a state of the art InGaAs channel pseudomorphic HEMT. We used an RF analysis technique based on transient Monte Carlo simulations, calibrated to a physical HEMT. The difference in device noise figures was attributed mainly to differences in device transconductance, and thus to differences in channel velocity. The cut-off frequency $f_{\mathrm{T}}$ was also shown to be governed by channel velocity. However the max- 
imum frequency of oscillation $f_{\max }$ was seen to exhibit a strong dependence on device contact resistance, eroding some of the advantage of the pseudomorphic HEMT over the Si/SiGe MODFET.

\section{References}

[1] Yamada, T., Zhou, J., Miyata, H. and Ferry, D. K. (1994). "In-Plane Transport Properties of $\mathrm{Si} / \mathrm{Si}_{1-x} \mathrm{Ge}_{x}$ Structure and its FET Performance by Computer Simulation", IEEE Trans. Elec. Devices, 41(9), 1513.

[2] Ismail, K., Rishton, S., Chu, J., Chan, K. and Meyerson, B. (1993). "High-performance $\mathrm{Si} / \mathrm{SiGe} n$-type modulationdoped transistors", IEEE Electron Device Letters, 14(7), $348-350$.

[3] Welser, J., Hoyt, J. L. and Gibbons, J. F. (1994). "Electron Mobility Enhancement in Strained-Si N-Type Metal-Oxide-Semiconductor Field-Effect Transistors", IEEE Electron Device Letters, 15(3), 100.

[4] Babiker, S., Asenov, A., Cameron, N., Beaumont, S. P and Barker, J. R. "Complete RF analysis of compound FETs based on transient Monte Carlo simulation", this issue.

[5] Jacoboni, C. and Lugli, P. (1989). The Monte Carlo Method for Semiconductor Device Simulation, Wein, Springer-Verlag.

[6] Brooks, H. (1951). "Scattering by Ionized Impurities in Semiconductors", Phys. Rev., 83, 879 .

[7] Roldén, J. B., Gámiz, F., López-Villanueva, J. A. and Carceller, J. E. (1996). "A Monte Carlo study on the electron-transport properties of high-performance strained-Si on relaxed SiGe channel MOSFETs", J. Appl. Phys., 80(9), 5121.

\section{Authors' Biographies}

Scott Roy is a researcher in the Department of Electronics and Electrical Engineering at the University of Glasgow, from where he received a Ph.D. in 1994 for investigations into the engineering and architectural aspects of extended single electronic systems. His interests include high performance computing (he represented Glasgow University in ESPRIT Project ZEUS; Zentren Europáischen Supercomputings) and its application to device modelling. He is presently developing codes to simulate and optimise $n$-channel SiGe FETs for VLSI and RF applications - and designing, in collaboration with Motorola UK, multiprocessor systems on which they will run.
Sherief Babiker studied Electrical Engineering in Khartoum University Sudan (1979-1984). During the period 1985-90 he worked at the Electrical Engineering Department of Khartoum University. In 1994 he was awarded a Ph.D. degree for work on the theory and modelling of single-electronic devices and systems. His current research interests concentrate on the simulation and modelling of FETs with emphasis on submicron gate length InGaAs channel pHEMTs for RF applications.

Asen Asenov had 10 years industrial experience as a head of the Process and Device Modelling Group in IME - Sofia, developing one of the first integrated process and device CMOS simulators IMPEDANCE. He was visiting professor at the Physics Department of TU Munich, and is currently a Reader in the Department of Electronics and Electrical Engineering, Glasgow University. As a leader of the Device Modelling Group he has contributed to the development of $2 \mathrm{D}$ and 3D device simulators and their application in the design of FETs, SiGe MOSFETs and IGBTs. He also investigates the design of parallel algorithrns.

John Barker is Professor of Electronics in the Department of Electronics and Electrical Engineering. He has a long standing interest in computational methods, device modelling and transport theory. From $1970-85$ he was a member of the Theory group in the Dept. of Physics, University of Warwick, aside from 1978-79 when he worked at IBM T.J. Watson Laboratory, North Texas State University and Colorado State University. From 1987-89 he was academic director of the IBM UK/Glasgow University Kelvin Project on Numerically Intensive Paraliel Computing. He is academic director of the Parallel Processing Centre at the University of Glasgow.

Steve Beaumont was educated at the University of Cambridge and has been with the Department of Electronics and Electrical Engineering at the University of Glasyow since 1978. He became Head of Department in 1995 and he convenes the Nanoelectronics Research Centre's management cornmittee. His research interests lie in the field of 
nanometre-scale fabrication and its application to electronic and optoelectronic devices. He has over 100 publications on electron beam nanolithography, dry etching, short-gate III - V based transistors, quantum transport devices, the optical properties of quantum dots, and single electron devices. Latterly he has become involved with the issue of manufacturability of $\mathrm{mm}$-wave circuits and the use of nanometre-scale fabrication techniques coupled with technology-based device simulations to forecast performance and yield with the minimum of process iterations. 

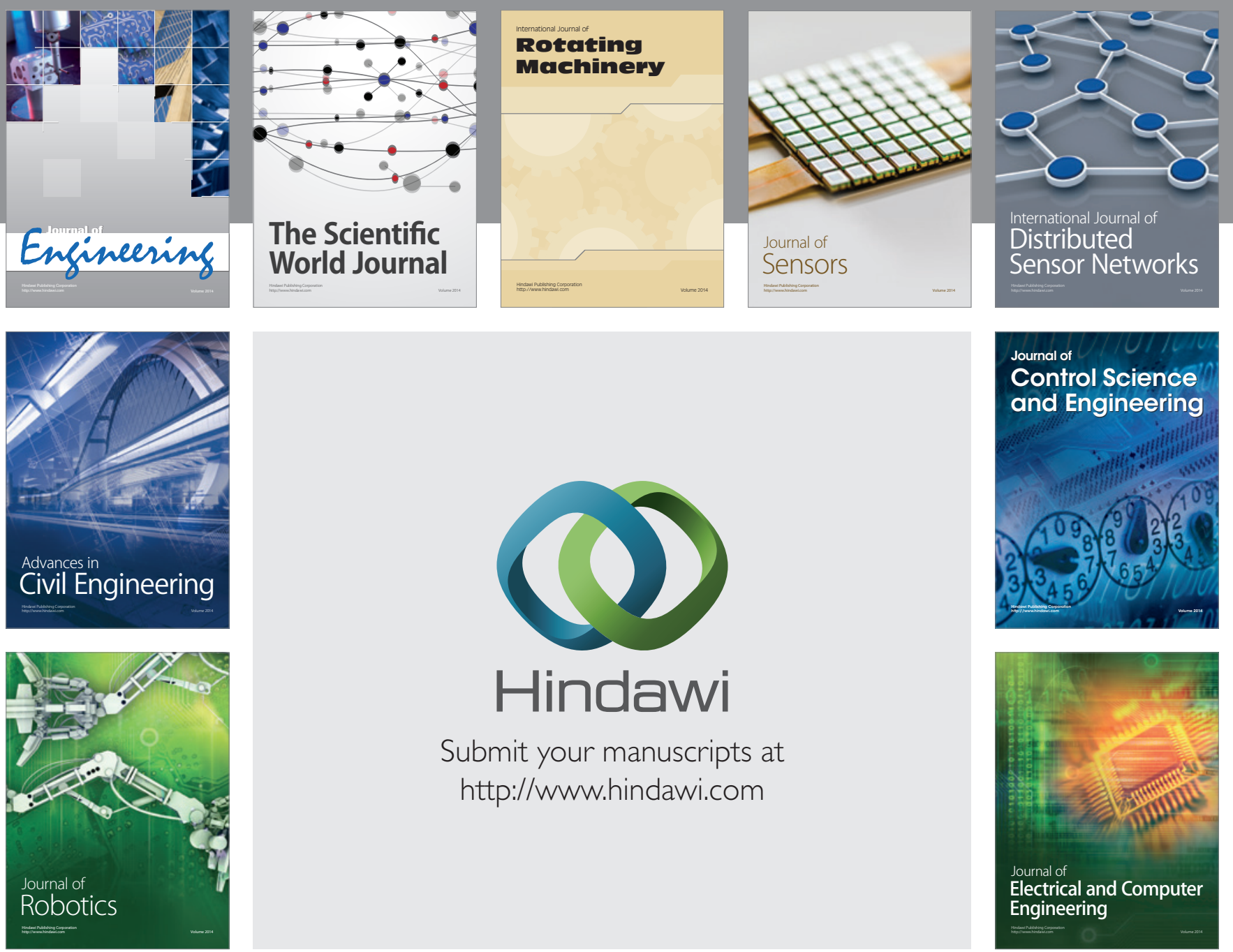

Submit your manuscripts at

http://www.hindawi.com
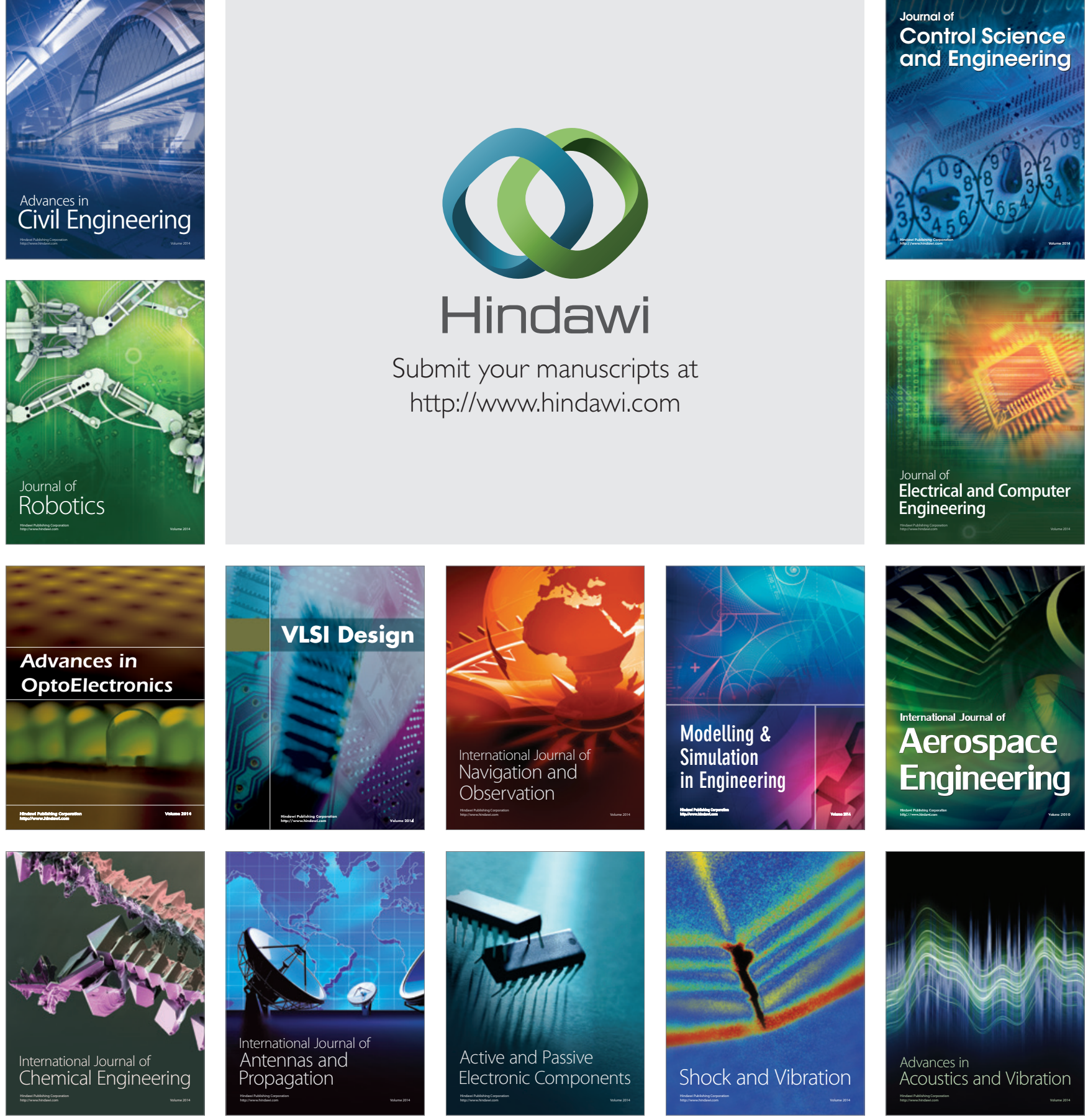